\title{
Pressure Dependence of the Roton Spectrum in Liquid Helium*
}

\author{
David L. .Bartley $\dagger$ \\ Department of Physics, University of Michigan, Ann Arbor, Michigan \\ John E. Robinson \\ Argonne National Laboratory, Argonne, Illinois \\ and Chia-Wei Woot \\ Department of Physics, Northwestern University, Evanston, Illinois
}

(Received November 26, 1973)

We suggest consistency checks on neutron scattering data on liquid helium under pressure. The theoretically calculated static structure function $\mathbf{S}(\mathbf{k} \mid \mathbf{P})$ leads to pressure dependence of the roton gap, momentum, and curvature in general agreement with experiment.

Recent experiments at Argonne, ${ }^{1}$ Brookhaven, ${ }^{2}$ and Chalk River ${ }^{3}$ indicate that the excitation spectrum of liquid helium undergoes significant shifts when subjected to external pressure. We point out in this note that certain well-known relations exist between the measured properties, and that these relations can be used as consistency checks on the experiment.

First, there are sum rules dealing with various moments of the dynamic structure function $S(k, \omega)$. These sum rules have been used ${ }^{4,5}$ in analyzing neutron and $x$-ray scattering properties at zero pressure. They are equally valid at higher pressures. However, as yet the available experimental data are neither detailed nor accurate enough to justify similar analyses.

Second, there exist perturbation formulas relating the excitation spectrum to the static structure function $S(k)$. In particular, the second-order

*Work supported in part by the U.S. Atomic Energy Commission, and in part by the National Science Foundation under Grant No. GP-29130 and through the Materials Research Center of Northwestern University.

$\nmid$ Resident Student Associate at Argonne National Laboratory, Summer 1972 and 1973.

¥Alfred P. Sloan Research Fellow. 
Brillouin-Wigner formula first employed by Jackson and Feenberg ${ }^{6}$ expresses the excitation energy $\varepsilon(k \mid \rho)$ in terms of integrals involving $S(k \mid \rho)$ for given density $\rho$. At a fixed temperature, say $T=0 \mathrm{~K}$, the equation of state determines the pressure $P$ corresponding to each $\rho$. We thus find a useful integral relation between $\varepsilon(k \mid P)$ and $S(k \mid P)$.

Finally, it is actually possible to carry out variational calculations for the ground state of liquid ${ }^{4} \mathrm{He}$. Such calculations give rise to zero-temperature $S(k \mid \rho)$, or alternatively $S(k \mid P)$, as was first demonstrated by Massey. ${ }^{7}$ The pressure dependence obtained can be compared directly with experiment. In the following paragraphs we elaborate on some of these remarks.

A natural extension of Feynman's theory of the phonon-roton spectrum to include interference effects results in wave functions in the form of admixtures of single-phonon and multiphonon states. In first approximation, one might consider the space spanned by one- and two-phonon states:

and

$$
|\mathbf{k}\rangle=\rho_{\mathbf{k}} \psi_{0}
$$

where

$$
|\mathbf{k}-\mathbf{l}, \mathbf{l}\rangle=\rho_{\mathbf{k}-1} \rho_{1} \psi_{0}
$$

$$
\rho_{\mathbf{k}} \equiv[N S(k)]^{-1 / 2} \sum_{i} \exp \left(i \mathbf{k} \cdot \mathbf{r}_{i}\right)
$$

represents density fluctuations operating on the ground state $\psi_{0}$. To obtain the optimum linear combination of $|\mathbf{k}\rangle$ and $|\mathbf{k}-1,1\rangle$, one minimizes the energy expectation value in the state

$$
\zeta_{\mathbf{k}}|\mathbf{k}\rangle+\sum_{\mathbf{l}} \xi_{\mathbf{k}}(\mathbf{l})|\mathbf{k}-\mathbf{I}, \mathbf{l}\rangle
$$

with respect to the weighting functions $\zeta_{k}$ and $\xi_{k}(1)$. This calls for the solution of coupled Euler-Lagrange equations, a procedure to be discussed elsewhere. ${ }^{8}$ It has been shown ${ }^{8}$ that (i) iterating the Euler-Lagrange equations results in a Brillouin-Wigner energy series, (ii) the series converges rapidly, and (iii) the omission of four-phonon processes described by the matrix elements $\langle\mathbf{k}-\mathbf{h}, \mathbf{h}|H| \mathbf{k}-\mathbf{l}, \mathbf{l}\rangle$ reduces the series to a much-simplified formula; the error introduced is small. The relation between $\varepsilon(k \mid \rho)$ and $S(k \mid \rho)$ to which we alluded is therefore given by ${ }^{6,9}$

$$
\begin{aligned}
\varepsilon(k \mid \rho)= & \varepsilon_{\mathrm{F}}(k \mid \rho)+\frac{\hbar^{2}}{16 m \pi^{2} \rho} \int_{0}^{\infty} q^{2} d q \int_{-1}^{+1} d \mu[\mathbf{k} \cdot(\mathbf{k}-\mathbf{q}) S(q) \\
& \left.+\mathbf{k} \cdot \mathbf{q} S(|\mathbf{k}-\mathbf{q}|)-k^{2} S(|\mathbf{k}-\mathbf{q}|) S(q)\right]^{2} \\
& \times[S(k) S(|\mathbf{k}-\mathbf{q}|) S(q)]^{-1} \\
& \times\left\{\frac{k^{2}}{S(k)}-\frac{|\mathbf{k}-\mathbf{q}|^{2}}{S(|\mathbf{k}-\mathbf{q}|)}-\frac{q^{2}}{S(q)}+\frac{2 m}{\hbar^{2}}\left[\varepsilon(k \mid \rho)-\varepsilon_{\mathrm{F}}(k \mid \rho)\right]\right\}
\end{aligned}
$$


with

$$
\varepsilon_{\mathrm{F}}(k \mid \rho) \equiv \frac{\hbar^{2} k^{2}}{2 m S(k \mid \rho)}
$$

denoting the Feynman spectrum.

We have not been able to obtain neutron scattering data on $S(k \mid P)$. Elastic $x$-ray scattering yields the static structure function directly. Plans are now under way ${ }^{10}$ to carry out these experiments under pressure; data will become available within the next year. In the meantime we resort to variational calculations of $S(k \mid \rho)$. This immediately rules out any sensible comparison at small momentum transfers since variational calculations are known to be insensitive with regard to the long-range behavior of the wave function. We thus have nothing to report concerning the pressure dependence of the sound velocity. On the other hand, the calculations are expected to be reasonably accurate at intermediate $k$-around the roton minimum. We are thus able to perform a theoretical check on the Brookhaven data: ${ }^{2}$

As shown in Figs. $1-3$ and Table II, Massey's $S(k \mid \rho)$ leads to results which are clearly unsatisfactory. We recalculate $S(k \mid \rho)$ over the entire range of liquid densities by means of a standard Jastrow procedure, which employs the trial wave function

$$
\psi_{0}(\rho)=\prod_{i<j} \exp \left[\frac{1}{2} u\left(r_{i j} \mid \rho\right)\right]
$$

with

$$
u(r \mid \rho)=-\left[\frac{a(\rho) \sigma}{r}\right]^{5}, \quad \sigma=2.556 \AA
$$

and $a(\rho)$ is to be determined variationally. The energy expectation value takes the form

$$
E(\rho)=\frac{1}{2} N \rho \int\left[v(r)-\left(\hbar^{2} / 4 m\right) \nabla^{2} u(r \mid \rho)\right] g(r \mid \rho) d \mathbf{r}
$$

where $v(r)$ is taken to be the deBoer-Michels potential, and $g(r \mid \rho)$ is the radial distribution function evaluated with the aid of the BBGKY equation:

$$
\begin{aligned}
& \nabla_{1} \ln g\left(r_{12} \mid \rho\right) \\
& =\nabla_{1} u\left(r_{12} \mid \rho\right)+\rho \int g\left(r_{23} \mid \rho\right) g\left(r_{13} \mid \rho\right) \nabla_{1} u\left(r_{13} \mid \rho\right) d r
\end{aligned}
$$

$g(r \mid \rho)$ is related to the static structure function via Fourier transformations; thus

$$
S(k \mid \rho)=1+\rho \int[g(r \mid \rho)-1] \exp (i \mathbf{k} \cdot \mathbf{r}) d \mathbf{r}
$$


TABLE I

Variationally Calculated Pressure-Dependent Liquid Structure Function

\begin{tabular}{|c|c|c|c|c|}
\hline \multirow[b]{2}{*}{$\rho, \AA^{-3}$} & \multirow[b]{2}{*}{$P$, atm } & \multirow[b]{2}{*}{$a(\rho)$} & \multicolumn{2}{|c|}{$S(k \mid \rho)$ Maximum } \\
\hline & & & Position, $\AA^{-1}$ & Height \\
\hline 0.02180 & 0.0 & 1.168 & 2.1 & 1.230 \\
\hline 0.02268 & 3.9 & 1.172 & 2.1 & 1.247 \\
\hline 0.02344 & 7.7 & 1.176 & 2.1 & 1.263 \\
\hline 0.02417 & 12.2 & 1.180 & 2.1 & 1.281 \\
\hline 0.02490 & 17.4 & 1.184 & 2.1 & 1.298 \\
\hline 0.02560 & 23.0 & 1.188 & 2.1 & 1.317 \\
\hline
\end{tabular}

Table I exhibits the main features of the variational results. Because of its two-particle nature, the wave function of Eq. (7) does not contain sufficient structure. The $S(k \mid \rho)$ peaks are too low and too broad. While it is in principle possible to improve on the Jastrow calculation by introducing three-particle correlations into the wave function, ${ }^{11}$ we believe that a rough estimate of the pressure dependence may be obtained from the present two-body correlation calculation. Making use of the zero-temperature equation of state, ${ }^{12}$ and expressing the pressure dependence of the position $k_{\max }(P)$ and height of the $S(k \mid P)$ peak in the following manner:

$$
\left\{\begin{aligned}
k_{\max }(P) & \approx k_{\max }(0)(1+\alpha P) \\
S\left(k_{\max }(P) \mid P\right) & \approx S\left(k_{\max }(0) \mid 0\right)(1+\beta P)
\end{aligned}\right.
$$

we find $|\alpha|<3 \times 10^{-3} \mathrm{~atm}^{-1}$ and $\beta=3.6 \times 10^{-3} \mathrm{~atm}^{-1}$. These coefficients are offered as predictions.

Substituting the calculated value of $S(k \mid \rho)$ into Eqs. (5) and (6) and solving them numerically, we find the pressure dependence of the roton parameters as shown in Figs. 1-3. Table II shows the variation with density $\rho$ of the gap $\Delta$, the roton momentum $k_{0}$, and the curvature parameter $\mu$.

The calculated roton minimum parameters as well as their pressure dependences show significant if semiquantitative agreement with experiment.

TABLE II

Pressure Dependence of Roton Parameters

\begin{tabular}{lccc}
\hline & $d \Delta / d \rho, k_{\mathrm{B}} \mathrm{K} \AA^{3}$ & $d k_{0} / d \rho, \AA^{2}$ & $d \mu / d \rho, m_{\mathrm{He}} \AA^{3}$ \\
\hline Experiment $^{2}$ & -380 & 29 & -9.4 \\
Present Theory & -220 & 36 & -22.0 \\
Massey & -16 & 34 & 6.3 \\
Henshaw & -270 & 29 & 9.3 \\
\hline
\end{tabular}


Fig. 1. Pressure dependence of the roton gap $\Delta$. (O) calculated using Massey's structure function (see Ref. 7). (O) Calculated by the present theory. $(\Delta)$ Calculated using the experimental structure function of Henshaw (see Ref. 13). ( $\mathbf{\Delta}$ ) experimental, Dietrich et al. (see Ref. 2). The lines are just aids to the eye and do not represent any calculation or best fit.
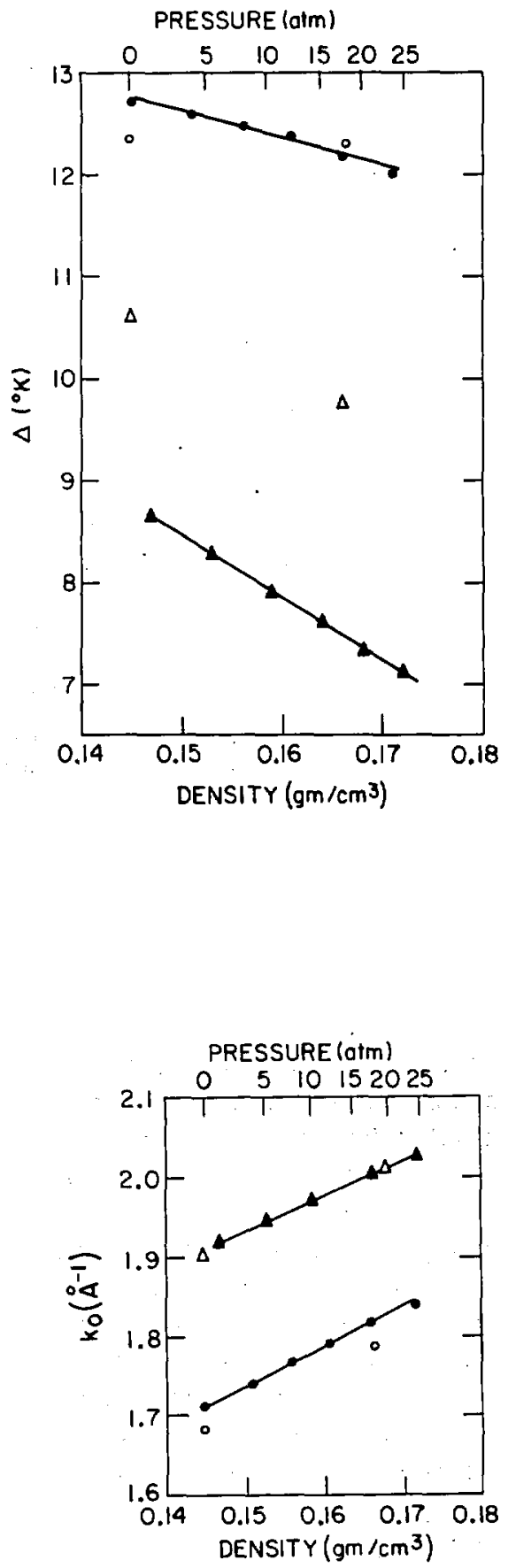

Fig. 2. Pressure dependence of the roton momentum $k_{0}$. Points and lines as for Fig. 1 . 


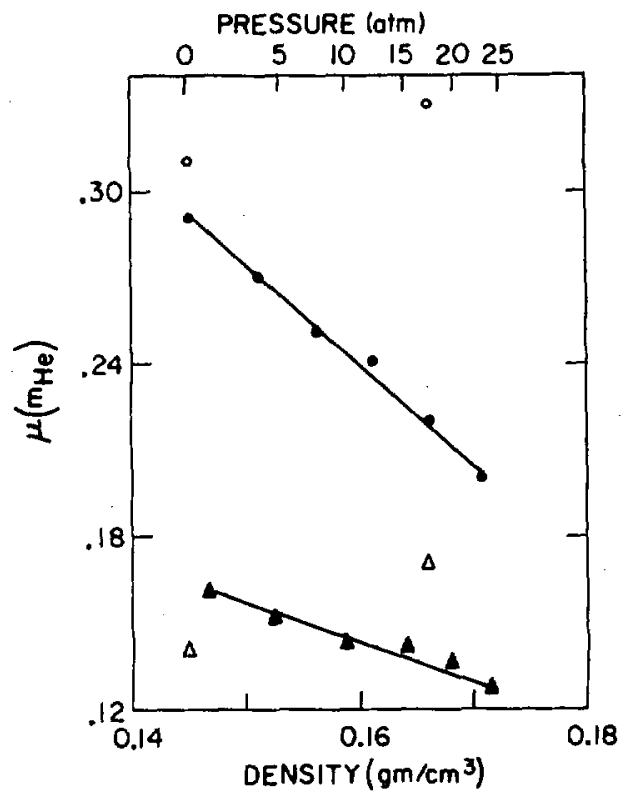

Fig. 3. Pressure dependence of the roton curvature parameter $\mu$. Points and lines as for Fig. 1.

We believe that the discrepancies concerning at least the roton energy gap and its pressure dependence arise primarily from our neglect of higher than two-body correlations in specifying the system's ground state. This is borne out by a previous calculation which showed that three-body correlations tend to raise and sharpen the major peak of the structure function. ${ }^{11}$ Equation (6) then implies that for a given pressure the roton minimum would be lowered, thus approaching the experimental value. Furthermore, the pressure dependence of the gap energy may also be enhanced. This follows from the fact that three-body correlations should become increasingly more important as the density is increased, and thus, according to the above argument, the rate of decrease of the gap energy with pressure increase should be greater than that calculated assuming only two-particle correlations.

It should be mentioned that the experimental $S(k)$ at $P=0$ has a notably sharper peak than the Jastrow result. This encourages us to anticipate that analysis of the type proposed here may indeed provide a consistent framework with which to relate experimentally determined $\varepsilon(k \mid P)$ and $S(k \mid P)$. In an attempt to assess this view, we include in this report results obtained with experimental $S(k \mid P)$ at two pressures. ${ }^{13 *}$ There is some tentative evidence of

*Note that there are just two data points: $P=0$ at $T=1.06 \mathrm{~K}$ and $P=14.9$ atm at $2.05 \mathrm{~K}$. 
improved consistency, but note that the higher pressure data were obtained at a higher temperature. Clearly, more experiments are needed.

\section{ACKNOWLEDGMENT}

We are grateful to Prof. Yu Ming Shih for his assistance in the computations.

\section{REFERENCES}

1. P. R. Roach, J. B. Ketterson, B. M. Abraham, and M. Kuchnir, J. Low Temp. Phys. 12, 375 (1973).

2. O. W. Dietrich, E. H. Graf, C. H. Huang, and L. Passell, Phys. Rev. A 5, 1377 (1972).

3. E. C. Svensson, A. D. B. Woods, and P. Martel, Phys. Rev. Lett. 29, 1148 (1972).

4. D. Pines and C.-W. Woo, Phys. Rev. Lett. 24, 1044 (1970); 25, 197 (1970).

S. R. A. Cowley and A. D. B. Woods, Can. J. Phys. 49, 177 (1971).

6. H. W. Jackson and E. Feenberg, Rev. Mod. Phys. 34, 686 (1962).

7. W. E. Massey, Phys. Rev. 151, 153 (1966).

8. Y. R. Lin-Liu and C.-W. Woo, to be published.

9. H.-W. Lai, H.-K. Sim, and C.-W. Woo, Phys. Rev. A 1, 1536 (1970).

10. R. B. Hallock, private communication.

11. C.-W. Woo and R. L. Coldwell, Phys. Rev. Lett. 29, 1062 (1972); C.-W. Woo, Phys. Rev. A 6, 2312 (1972).

12. B. M. Abraham, Y. Eckstein, J. B. Ketterson, M. Kuchnir, and P. R. Roach, Phys. Rev A 1, 250 (1971).

13. D. G. Henshaw, Phys. Rev. 119, 9, 14 (1960). 